\title{
Bloqueos de Extremidad Superior y Cirugía Ambulatoria: Revisión de la Evidencia Actual
}

\author{
Upper extremity nerve blocks for Outpatient surgery: A summary of current evidence. \\ https://doi.org/10.25237/carsach2020.12
}

Dra. Andrea Araneda V. ${ }^{1}$ Dr. Pablo Miranda H. ${ }^{1}$ Dra. Marcia Corvetto A. ${ }^{1}$ Dr. Fernando Rueda B. ${ }^{1}$ Dr. Fernando Altermatt C. ${ }^{1}$

${ }^{1}$ División de Anestesiología. Escuela de Medicina. Pontificia Universidad Católica de Chile.

Autor Corresponsal:

Dra. Andrea Araneda V.

División de Anestesiología. Escuela de Medicina. Pontificia Universidad Católica de Chile. andreaaraneda10@gmail.com

ORCID ID: https://orcid.org/0000-0002-8375-0653

Palabras claves:

Bloqueo de Plexo braquial, Bloqueo de Nervio periférico, Extremidad Superior, Anestesia Regional, Cirugía Ambulatoria.

Key words:

Brachial Plexus block, Peripheral Nerve blocks, Upper Extremity, Regional Anesthesia, Outpatient Surgery.

\section{Puntos Claves}

- La anestesia regional proporciona una mejora significativa de la analgesia postoperatoria y calidad de la recuperación en la cirugía ortopédica ambulatoria.

- El uso de los bloqueos de nervio periférico continuo extiende sus beneficios más allá de la duración del bloqueo de punción única con escasas complicaciones.

- Las cirugías de extremidad superior se pueden realizar de manera segura con anestesia regional o combinada con anestesia general o sedación con una excelente analgesia postoperatoria.

- La elección del bloqueo a realizar dependerá del paciente y sus comorbilidades, sitio quirúrgico, tipo de cirugía , experiencia del anestesiólogo y características del establecimiento de Salud.

- La anestesia regional para cirugía ambulatoria es un desafío constante para el equipo, dado su crecimiento, incorporación de técnicas nuevas así como también de la necesidad de evaluación constante de los resultados y complicaciones de los pacientes.

\section{Resumen:}

La Cirugía ambulatoria ha aumentado durante las últimas décadas, así como también el uso de anestesia regional. Los beneficios de la anestesia regional en el escenario ambulatorio son mejorar la calidad de analgesia, disminución del consumo de opioides, disminución de náuseas y vómitos en el postoperatorio, disminución en la estadía en recuperación y mayor satisfacción usuaria. 
En cuanto a las cirugías de extremidad superior ambulatorias, la cirugía artroscópica de manguito rotador es una de las cirugías más frecuentes. El bloqueo interescalénico es el gold standard para el manejo analgésico postoperatorio, sin embargo, el bloqueo ipsilateral del nervio frénico y la consecuente parálisis hemidiafragmática (PHD) limitan su uso en pacientes con compromiso pulmonar preexistente. En los últimos años, se han realizado múltiples esfuerzos con el fin de disminuir el riesgo de PHD, investigándose alternativas como el bloqueo supraclavicular, el bloqueo de tronco superior, el costoclavicular, el supraescapular y el axilar.

\begin{abstract}
:
Outpatient Surgery has increased during the last decades, as well as the use of regional anesthesia. The benefits of regional anesthesia in the outpatient setting includes better quality of analgesia, decreased opioid requirements, decreased post-operative nausea and vomiting, reduced stay in recovery room and greater patient satisfaction.
\end{abstract}

Regarding outpatient upper limb surgeries, arthroscopic rotator cuff surgery is one of the most common surgeries. Interscalene block is the gold standard for postoperative analgesic management, however, ipsilateral phrenic nerve block and the consequent hemidiaphragmatic paralysis (HDP) limit its use in patients with preexisting pulmonary disease. In recent years, multiple efforts have been made to reduce the risk of HDP, investigating alternatives such as supraclavicular block, upper trunk block, costoclavicular, suprascapular and axillary block.

This article summarizes the current evidence for regional anesthesia and its application to the most common surgeries of the upper extremity in outpatient surgery. This review concludes that regional anesthesia in this area is a contribution in current clinical practice but it requires further studies to enhance its benefits and reduces associated risks.

\title{
Introducción
}

La transición de procedimientos quirúrgicos desde los hospitales a la cirugía ambulatoria se incrementa cada año, dado la adopción de cirugías menos invasivas y del avance en técnicas anestésicas. La cirugía ambulatoria ha ido aumentando en las últimas décadas, actualmente sobre el 50\% de procedimientos quirúrgicos en Estados Unidos se realizan en pacientes ambulatorios. ${ }^{1}$ La anestesia regional ha demostrado ser beneficiosa en el escenario ambulatorio ya que ha mejorado la analgesia y disminuido las complicaciones como náuseas y vómitos logrando como resultado una menor estadía en la Unidad de Recuperación, menores tasas de readmisión hospitalaria y mayor satisfacción usuaria. $^{2-7}$

Se ha visto que la prevalencia de bloqueos regionales en cirugías ambulatorias desde el 2010 al 2015 utilizando la base de datos National Anesthesia Clinical Outcomes Registry (NACOR) es alrededor del 3,3\%. Si bien es relativamente baja, ha experimentado un aumento sostenido: el bloqueo del plexo braquial es el más frecuente con un $6,1 \%$ y la cirugía en la que ha aumentado más el uso de bloqueos es la artroscopia de hombro (41\%) seguido de la reconstrucción de ligamentos cruzados (32\%). ${ }^{8}$

En el contexto específico de cirugía de hombro ambulatorio, un reciente estudio de cohorte realizado por Hamilton en 59.644 pacientes desde el 2009 al 2016, reportó el uso de bloqueos de nervio periférico en el 52,1\% de los casos, destacando un incremento significativo de la incidencia con un 9,7\% en 2009 y sobre el $60 \%$ en los años 2015-2016. ${ }^{9}$ En relación a los costos, se encontró mayor gasto asociado al bloqueo, lo que sería estadísticamente significativo pero sin diferencia clínicamente relevante. ${ }^{9}$

Las indicaciones de bloqueos de la extremidad superior dependerán del sitio y tipo de cirugía, ${ }^{10}$ así como también del objetivo analgésico o anestésico según sea el caso, permitiendo reducción de consumo de opioides e incluso evitando anestesia general y sus efectos adversos. Las cirugías de la extremidad superior permiten la utilización de bloqueos de nervio periférico, principalmente dado que toda su inervación depende del plexo braquial, pudiéndose bloquear fácilmente con una inyección única (Tabla 1). ${ }^{11}$

El objetivo de esta revisión es hacer un análisis de la evidencia actual de los bloqueos de extremidad superior en la cirugía ambulatoria, en términos de analgesia y efectos colaterales. 


\section{Cirugía de Hombro}

La cirugía artroscópica de hombro se realiza como cirugía ambulatoria en la mayoría de los centros hospitalarios de Estados Unidos, siendo la reparación artroscópica del manguito rotador la cirugía más frecuente. ${ }^{8}$ La cirugía de hombro está asociada a tres veces más ingresos no anticipados versus otras cirugías traumatológicas ambulatorias. ${ }^{12} \mathrm{La}$ causa específica para esta asociación aún no ha sido dilucidada. Sin embargo, se sabe que la cirugía de hombro es una de las cirugías más dolorosas ${ }^{12-14}$ en el postoperatorio, tanto como la toracotomía o artroplastía de rodilla. ${ }^{15,16}$

Si bien los beneficios de la anestesia regional en la cirugía ambulatoria de hombro en cuanto a dolor han sido demostrados, en un reciente estudio de cohorte retrospectivo no se observó diferencias en admisiones no planificadas, readmisiones a los 30 días, o visitas al servicio de urgencias dentro de los 30 días post cirugía. ${ }^{17}$

La readmisión perioperatoria (a 30 días) de cirugías ortopédicas ambulatorias es del $5 \% .{ }^{18-20}$ Es por esto, que la cirugía artroscópica de hombro continúa siendo un desafío donde es muy importante una adecuada analgesia postoperatoria que permita una recuperación rápida dentro del contexto ambulatorio. Es así como la anestesia regional ha logrado que estos pacientes alcancen criterios de alta precozmente y reporten mayor satisfacción usuaria. ${ }^{21}$

El bloqueo interescalénico (IE) continúa siendo el gold standard para la analgesia de la cirugía de hombro. ${ }^{22,23}$ Lamentablemente no todos los pacientes califican para este bloqueo, principalmente por el bloqueo del nervio frénico ipsilateral y la parálisis hemidiafragmática (PHD). Previo al uso del Ultrasonido y asociado a los grandes volúmenes de anestésicos locales utilizados, el riesgo de PHD asociada al bloqueo IE era del $100 \% .{ }^{24,25}$ Con el ultrasonido se ha podido utilizar menores volúmenes $(5 \mathrm{ml})^{26-28}$, concentraciones más diluidas (bupivacaina $0,125 \%$, ropivacaína $0,1 \%)^{29,30}$ y punciones extrafasciales del plexo braquial, ${ }^{31}$ logrando incidencias de PHD de un $\mathbf{2 0 \%}$. $^{32}$ Aún así, en los últimos años se ha tratado de buscar alternativas al bloqueo IE con el fin de evitar dicho efecto, en particular en la población de riesgo de presentar falla respiratoria. El bloqueo supraclavicular es el más investigado; otros bloqueos estudiados son el bloqueo de tronco superior, el bloqueo del nervio supraescapular y bloqueo del nervio axilar. ${ }^{33}$

En cuanto al uso del bloqueo IE continuo en el contexto ambulatorio, la instalación y uso del catéter es técnicamente desafiante ${ }^{15}$ tanto para el equipo de anestesiólogos como quirúrgico y a veces se subutiliza por el temor a problemas logísticos y a la posibilidad de complicaciones fuera del hospital. ${ }^{34}$ A pesar de estos temores, las complicaciones son bajas. Fredrickson analizó 1505 pacientes con bloqueo IE continuo y encontró que un 27\% de los pacientes reportaron disnea (todos de manejo ambulatorio sin necesidad de hospitalización), 13\% ronquera, 7\% disfagia. Dentro de las complicaciones del catéter, se reportó desplazamiento en un $1,5 \%$, filtración $8 \%$, y problemas con la bomba $2 \%$. ${ }^{35}$ En otra revisión, donde se analizaron 509 pacientes con bloqueo IE continuo, se identificaron un $6,7 \%$ de eventos adversos donde lo más común fue analgesia insuficiente, 2,2\% desplazamientos del catéter y un 2,4\% de readmisión hospitalaria, principalmente por dolor. ${ }^{36}$

Se sabe que la analgesia de un bloqueo regional puede prolongarse en el tiempo ya sea con el uso de un bloqueo continuo, con drogas coadyuvantes o con anestésicos locales de larga duración, como la bupivacaína liposomal la cual está aprobada por la FDA (Food And Drug Administration) ${ }^{37}$ para su utilización en el bloqueo IE, pero que no está disponible en nuestro país.

En el caso de la cirugía de hombro artroscópica de manguito rotador se recomienda el bloqueo IE continuo por sobre la inyección única, ${ }^{23}$ principalmente por la duración de la analgesia y para evitar el dolor de rebote, ${ }^{38}$ definido como dolor agudo postoperatorio que aparece después de la resolución de un bloqueo de nervio periférico y que es clínicamente significativo que suele aparecer antes de las 24 horas lo que en un ambiente ambulatorio puede ser causa de readmisión del paciente. ${ }^{39}$ 
Por otro lado, como un esfuerzo por prolongar la duración de la inyección única del bloqueo IE, se han administrado distintos coadyuvantes perineurales combinados con el anestésico local. Actualmente hay una evidencia limitada de beneficios del uso perineural de buprenorfina, ${ }^{40}$ tramadol $^{41}$ y sulfato de magnesio, ${ }^{42}$ no hay evidencia que avale el uso perineural de clonidina ${ }^{43}$ y en cuanto a corticoides existe evidencia de beneficio perineural e intravenoso. ${ }^{44-46}$, es importante recordar que el uso de drogas perineurales continua siendo off-label. ${ }^{47}$ En la revisión PROSPECT de cirugía de manguito rotador recomiendan el uso de la dexametasona como dosis única intravenosa para aumentar la duración de la analgesia del bloqueo IE, disminuir uso de analgésicos y por su efecto antiemético (Tabla 2). 23,48

\section{Bloqueo Supraclavicular}

Actualmente la mayoría de los estudios sugieren que el bloqueo supraclavicular comparado con el bloqueo IE logra un similar control del dolor, consumo de analgésicos y satisfacción de los pacientes. ${ }^{49-51}$ A pesar de esto, igual se ve una PHD mayor al $60 \%$ cuando la punción se realiza intra cluster. ${ }^{50}$ Aliste et al, comparó bloqueo IE con el bloqueo supraclavicular, depositando el anestésico local en el corner pocket y postero-lateral al plexo encontrando una tasa de PHD de $9 \%$ y una analgesia equivalente al bloqueo IE para cirugía artroscópica de hombro. ${ }^{51}$

\section{Bloqueo de Tronco Superior}

El tronco superior está formado por la unión de las raíces C5 y C6, considerando que los nervios terminales que inervan el hombro emergen distal al tronco superior, con un bloqueo a este nivel la eficacia analgésica no se ve comprometida ${ }^{52}$. En el 2019, se realizó un estudio randomizado controlado comparando bloqueo IE y bloqueo de tronco superior para cirugía de hombro, donde se vio puntuaciones de dolor no inferiores en la Unidad de Recuperación y consumo similar de opioides en ambos grupos. ${ }^{53}$ Aún así, hay proximidad con el nervio frénico con una incidencia de PHD del 5\%, dado estos efectos respiratorios se tiende a elegir bloqueos más distales con el fin de reducir estos efectos.

\section{Bloqueo Supraescapular y Axilar}

En cirugía artroscópica de hombro, el bloqueo del nervio supraescapular o combinado con bloqueo del nervio axilar ha mostrado mejor calidad de analgesia comparado con placebo o infiltración local subacromial, pero menor efectividad al compararlo con el bloqueo IE.

En vista de la menor calidad analgésica estos bloqueos debieran reservarse para pacientes de riesgo de presentar hipoxemia o disnea sintomática significativa frente a la hemiparálisis diafragmática, como son pacientes con disfunción respiratoria preexistente u otras comorbilidades como la obesidad ${ }^{52}$. En un estudio multicéntrico aleatorizado realizado este año, que comparó inyecciones únicas de bloqueo supraescapular anterior con bloqueo IE, muestra que el bloqueo supraescapular no fue inferior en el manejo de dolor postoperatorio a las 24 horas y que consistentemente hubo bloqueo del tronco superior, por lo que se plantea como una alternativa eficiente al bloqueo IE. ${ }^{54}$ Con respecto al bloqueo supraescapular posterior, hay varios ensayos clínicos aleatorizados que concluyen que es inferior al bloqueo IE en el control del dolor al menos en las primeras 4 horas post quirúrgicas en la cirugía artroscópica de hombro lo que retrasa el alta en pacientes ambulatorios. ${ }^{55-57}$ Aún faltan estudios que permitan cuantificar el riesgo de PHD en el bloqueo supraescapular anterior.

\section{Bloqueo Costoclavicular}

Bloqueo descrito el año 2015, que aborda el plexo braquial en la fosa infraclavicular proximal, donde los tres cordones están estrechamente agrupados. En un RCT que comparó el bloqueo IE con el bloqueo costoclavicular, no se encontró diferencias en puntajes de dolor postoperatorio, consumo de opioides y satisfacción usuaria en las primeras 24 horas. Destaca que en este trabajo hubo 0\% de PHD en el bloqueo costoclavicular. Aun faltan estudios para confirmar estos resultados. 58

\section{Cirugía de Codo, Antebrazo y Mano}


Los bloqueos que más se utilizan en este tipo de cirugías son el bloqueo supraclavicular, infraclavicular y axilar. Su elección dependerá del tipo y localización de la cirugía, experiencia del anestesiólogo y características del paciente. ${ }^{11}$ El bloqueo supraclavicular, es un bloqueo que se realiza a nivel de troncos y/o divisiones del plexo braquial, y es donde el plexo se encuentra más compacto. Es por esto que es un bloqueo de latencia corta y una distribución bastante homogénea, cuando se realiza la punción intracluster (PHD de hasta un 60\%) y como técnica anestésica. ${ }^{50,59}$ La incidencia de neumotórax ha disminuido con el uso del ultrasonido lo que en los últimos años ha incentivado su uso. ${ }^{60}$ Principalmente utilizado para cirugías de toda la extremidad superior exceptuando el hombro y la región medial del brazo que es inervada por el nervio intercostobraquial.

El bloqueo Infraclavicular aborda el plexo a nivel de los cordones. Por sus características anatómicas es un bloqueo que permite el uso de catéteres para infusión continua. Principalmente utilizado en cirugías distales a la diáfisis humeral. Estudios que han comparado este bloqueo como técnica anestésica versus anestesia general en cirugía ambulatoria han encontrado que el uso del bloqueo disminuye la estadía en la Unidad de Recuperación, presenta mejor analgesia, menor uso de analgésicos, y mayor satisfacción usuaria. ${ }^{4}$

En cuanto a la hemiparálisis diafragmática se ha visto una incidencia del $3 \%$ con uso de ultrasonido. ${ }^{61}$ Un metaanálisis realizado el 2017 que comparó bloqueo infraclavicular con bloqueo supraclavicular encontró un bloqueo sensitivo más incompleto en territorio radial a los 30 minutos en el primero, lo que se evita con técnica de doble o triple punción; por otro lado no encontró diferencias en éxito de bloqueo, tiempo de ejecución, latencia y duración de analgesia. $^{62}$

En la cirugía de codo ambulatoria, la evidencia es limitada, un estudio aleatorizado controlado realizado el 2018 que comparó bloqueo infraclavicular con supraclavicular, encontró que ambas técnicas regionales son efectivas en este tipo de cirugías y comparables en tiempos de inicio de acción y de ejecución del procedimiento. La única diferencia encontrada fue una mayor frecuencia de parestesia durante la inserción de la aguja en el bloqueo supraclavicular ( $23 \%$ vs $8,3 \%$ en el bloqueo infraclavicular). ${ }^{63}$ Chin et al, publicó una revisión sistemática Cochrane, del uso del bloqueo infraclavicular en cirugía de extremidad superior distal. Basado en 15 estudios, concluye que el bloqueo infraclavicular es una efectiva alternativa al bloqueo supraclavicular y al bloqueo axilar, con una duración de analgesia postoperatoria similar a éstos, además de otorgar un mejor control del dolor ante el uso del torniquete comparado con el bloqueo axilar y menor tiempo de ejecución comparado con la multi-inyección del bloqueo axilar. ${ }^{64} \mathrm{El}$ bloqueo infraclavicular continuo presenta ventajas ya que dado su anatomía y facilidad de fijación, limita el desplazamiento y salida del catéter y mantiene condiciones de esterilidad, lo que es importante en la población de pacientes ambulatorios. 65

La evidencia existente al comparar los abordajes infraclaviculares paracoracoideo y costoclavicular para cirugía de extremidad superior bajo el codo es escasa y controversial aún, un estudio no encontró diferencias en tiempo de inicio de acción del bloqueo, tiempo de ejecución y éxito de éstos. ${ }^{66}$ Otro estudio encontró un inicio más rápido del bloqueo sensitivo y un menor tiempo para poder comenzar con la cirugía con la versión costoclavicular (10 versus 20 minutos). ${ }^{67}$

El bloqueo axilar, bloquea las ramas terminales del plexo braquial. Ampliamente usado para cirugías más distales o selectivas de antebrazo y mano, hay que considerar la alta variabilidad anatómica y la necesidad del bloqueo del nervio musculocutáneo en el vientre del músculo coracobraquial. ${ }^{68}$ Es un bloqueo de bajo riesgo y pocas complicaciones al compararlo con bloqueos más proximales. ${ }^{69}$ La multi-inyección mejora la tasa de éxito del bloqueo, en términos de adecuada anestesia y bloqueo motor al compararlo con inyección doble o única usando neuroestimulación. ${ }^{70}$ El ultrasonido en el bloqueo axilar ha mejorado la tasa de éxito y reducido el tiempo de latencia de éste. $^{71,72}$ 
Un estudio realizado en pacientes con fractura de muñeca donde compara anestesia regional versus anestesia general frente a la necesidad de atención médica postoperatoria no planificada, muestra que en un total de 195 pacientes, la incidencia de visita médica no planificada a las 48 horas fue mayor en el grupo de bloqueo del plexo braquial inyección única que en el grupo de anestesia general (12\% vs 4\%). ${ }^{73}$ Adicionalmente, en el grupo bloqueo del plexo braquial inyección única hubo más consultas por dolor (20\% vs 5\%) y fue más frecuente el reporte de dolor severo en el postoperatorio ( $41 \%$ vs $10 \%)$. Estos hallazgos hacen ver la importancia de dar claras indicaciones a los pacientes en cuanto a la analgesia multimodal y de rescate, y por otra parte realizar una adecuada selección de pacientes que van a requerir bloqueos continuos o uso de coadyuvantes según la disponibilidad y experiencia del centro.

\section{Conclusiones}

La cirugía ambulatoria continúa experimentando un crecimiento sostenido lo que requiere de múltiples esfuerzos y revisión del funcionamiento e implementación actual, tanto del trabajo de los equipos como de las técnicas de Anestesia Regional.

El uso de anestesia regional para extremidad superior ha aumentado y se utiliza de forma frecuente sobretodo en la cirugía artroscópica de hombro, que es donde se encuentra mayor evidencia, logrando reducir el dolor, consumo de opioides, náuseas y vómitos postoperatorios, además de permitir el alta precoz y mejorar la satisfacción usuaria.

En cuanto a la readmisión de pacientes y costos, estudios recientes no han encontrado diferencias en pacientes sometidos a anestesia regional por lo que aún falta mayor evidencia.

El bloqueo interescalénico continuo es el gold standard para el manejo del dolor de la cirugía de hombro, pero es importante considerar las posibles complicaciones, realizar una adecuada selección de pacientes y ofrecer alternativas en caso de que no sea posible su ejecución. En este contexto, actualmente hay una amplia línea de investigación buscando alternativas regionales efectivas y comparables, así como también uso de coadyuvantes que permitan prolongar la duración de un bloqueo de punción única, sobretodo en centros que no disponen de unidades de dolor domiciliario.

Finalmente, es muy importante contemplar los beneficios de la anestesia regional dentro de un manejo multimodal del dolor en la cirugía ambulatoria, educando y dando claras indicaciones a los paciente por parte de todos los equipos involucrados.

Tabla 1. Técnicas de Anestesia Regional para Extremidad Superior

\begin{tabular}{|c|c|c|c|c|}
\hline Técnica & $\begin{array}{l}\text { Nivel de } \\
\text { Bloqueo }\end{array}$ & $\begin{array}{l}\text { Nervios Periféri- } \\
\text { cos Bloqueados }\end{array}$ & Aplicación Quirúrgica & Comentario \\
\hline Interescalénico & $\begin{array}{l}\text { Troncos superi- } \\
\text { ores y medios }\end{array}$ & $\begin{array}{l}\text { Bloqueo del plexo } \\
\text { braquial, pero bloqueo } \\
\text { inconsistente de tronco } \\
\text { inferior (nervio ulnar) }\end{array}$ & $\begin{array}{l}\text { Cirugía de hombro, } \\
\text { humero proximal y } \\
\text { medio }\end{array}$ & $\begin{array}{l}\text { Paresia del frénico hasta en } \\
100 \% \text { de los casos. Inade- } \\
\text { cuado para pacientes que } \\
\text { toleren menos del } 25 \% \text { de } \\
\text { reducción de la función pul- } \\
\text { monar }\end{array}$ \\
\hline Supraclavicular & $\begin{array}{l}\text { Troncos Distales } \\
\text {-cordones proxi- } \\
\text { males }\end{array}$ & $\begin{array}{l}\text { Radial, ulnar, mediano, } \\
\text { musculocutáneo, axilar }\end{array}$ & $\begin{array}{l}\text { Cirugía de húmero } \\
\text { medio, codo, antebrazo } \\
\text { y mano }\end{array}$ & $\begin{array}{l}\text { Riesgo de neumotórax y pare- } \\
\text { sia del frénico }\end{array}$ \\
\hline Infraclavicular & Cordones & $\begin{array}{l}\text { Radial, ulnar, mediano, } \\
\text { musculocutáneo, axilar }\end{array}$ & $\begin{array}{l}\text { Cirugía de codo, ante- } \\
\text { brazo y mano }\end{array}$ & $\begin{array}{l}\text { Menor riesgo de neumotórax } \\
\text { Adecuado para catéter }\end{array}$ \\
\hline Axilar & $\begin{array}{l}\text { Nervios Periféri- } \\
\text { cos }\end{array}$ & Radial, ulnar, mediano & $\begin{array}{l}\text { Cirugía de mano y ante- } \\
\text { brazo, menos usado } \\
\text { para cirugías cercanas } \\
\text { al codo. }\end{array}$ & $\begin{array}{l}\text { Inadecuada para cirugías } \\
\text { proximales. } \\
\text { Requiere abducción del brazo }\end{array}$ \\
\hline
\end{tabular}

Adaptado de Kopp SL. Regional Anaesthesia in day-stay and short-stay surgery. Anaesthesia 2010;65-Suppl1:84-96 11 
Tabla 2. Recomendaciones del manejo de dolor in pacientes operados de manguito rotador

Manejo pre e intraoperatorio

- Paracetamol (Grado D)

- Inhibidores COX-2 (Grado D)

- Dexametasona i.v. (Grado B)

- Analgesia Regional

- Bloqueo Interescalénico Continuo (Grado A)

- Bloqueo Interescalénico Punción única (Grado A)

- Bloqueo Supraescapular con o sin bloqueo de nervio axilar (no como primera opción, Grado B)

Manejo Postoperatorio

- Paracetamol (Grado D)

- Inhibidores COX-2 /AINEs (Grado D)

- Rescate de Opioides (Grado D)

COX, ciclo-oxigenasa. AINEs, antiinflamorios no esteroidales. Adaptado de Toma O, Persoons B, PogatzkiZahn E, Van de Velde M and Joshi GP on behalf of the PROSPECT Working Group collaborators. Anaesthesia 2019;74:1320-1331. ${ }^{23}$

\section{Referencias}

1.- Wier LM, Steiner CA, Owens PL. Surgeries in hospital-owned outpatients facilities, 2012, 2015. https:// www.hcup-us.ahrq.gov/reports/statbriefs/sb188-Surgeries-Hospital-Outpatiente-Facilities-2012-pdf.

2.- Ilfeld BM. Continuous peripheral nerve blocks: an update of the published evidence and comparison with novel, alternative analgesic modalilities. Anesth Analg. 2017;124:308-335.

3.- Lin E, Choi J, Hadzic A. Peripheral nerve blocks for outpatient surgery: evidence-bases indications. Curr Opin Anaesthesiol.2013;26:467-474.

4.- Hadzic A, Arliss J, Kerimoglu B, et al. A comparison of infraclavicular nerve block versus general anesthesia for hand and wrist day-case surgeries. Anesthesiology 2004;101:127-132.

5.- Hadzic A, Karaca PE, HobeikaP, et al. Peripheral nerve blocks result in superior recovery profile compared with general anesthesia in outpatient knee arthroscopy. Anesth Analg.2005;100:976-981.

6.- Hadzic A, Kerimoglu B, Loreio D, et al. Paravertebral blocks provide superior same day recovery over general anesthesia for patients undergoing inguinal hernia repair. Anesth Analg.2006;102:1076-1081.

7.- Chan EY, Fransen M, Parker DA. Femoral nerve blocks for acute postoperative pain after knee replacement surgery. Cochrane database syst rev 2014;5:Cd009941.

8.- Rodney G, Ilfeld BM. Use of regional anesthesia for outpatient surgery within the United States: A prevalence study using a Nationwide database. Anesth Analg 2018; 126:2078-84.

9.- Hamilton GM, Ramlogan R, Lui A, et al. Peripheral nerve blocks for ambulatory shoulder surgery: a populationbased cohort study of outcomes and resource utilization. Anesthesiology 2019;131:1254-63.

10.- Coleman AC. Perioperative pain management for upper extremity surgery. Orthop Clin North Am 2017;48:487-94.

11.- Kopp SL, Horlocker T. Regional Anaesthesia in day-stay and short-stay surgery. Anaesthesia 2010;65Supp11:84-96

12.- Memtsoudis SG, Ma Y, Swamidoss CP. Factors influencing unexpected disposition after orthopedic ambulatory surgery. J Clin Anesth 2012;24:89-95. 
13.- Fortier J, Chung F, Su J. Unanticipated admission after ambulatory surgery-a prospective study. Can J Anaesth 1998;45:612-9.

14.- Gerbershagen HJ, Aduckathil S, Van Wijck A. Pain intensity on the first day after surgery: a prospective cohort study comparing 179 surgical procedures. Anesthesiology 2013;118:934-44.

15.- Lindberg MF, Grow EK, Gay Cl, et al. Pain characteristics and self-rated health after elective orthopaedic surgery-a cross-sectional survey. J Clin Nurs 2013;22:1242-53.

16.- Fredrickson MJ, Krishnan S, Chen CY. Postoperative analgesia for shoulder surgery: a critical appraisal and review of current techniques. Anaesthesia 2010; 65:608-24.

17.- Hamilton G, Ramlogan R, Lui A. Association of peripheral nerve blocks with postoperative outcomes in ambulatory shoulder surgery patients: a single-centre matched-cohort study. Can J Anaesth 2019;66:63-74.

18.- Bernatz J, Tueting J, Anderson P. Thirty-day readmission rates in orthopedics: a systematic review and metaanalysis. PloS One 2015; 10:e123593.

19.- Mahoney A, Bosco J, Zuckerman J. Readmission after shoulder arthroplasty. J Shoulder Elbow Surg 2014;23:377-81.

20.- Sherman S, Lyman S, Koulouvaris P. Risk factors for readmission and revision surgery following rotator cuff repair. Clin Orthop Relat Res 2008;466:608-13.

21.- D’ Alessio JG, Rosenblum M, Shea KP. A retrospective comparison of interscalene block and general anesthesia for ambulatory surgery shoulder arthroscopy- Reg Anesth 1995;20:62-68.

22.- Abdallah FW, Halpern SH, Aoyama K, et al. Will the real benefits of single-shot interescalene block please stand up? A systematic review and meta-analysis. Anesth Analg 2015;120:114-29.

23.- Toma O, Persoons B, Pogatzki-Zahn E, Van de Velde M. PROSPECT guideline for rotator cuff repair surgery: systematic review and procedure-specific postoperative pain management recommendations. Anaesthesia 2019;74:1320-1331.

24.- Urmey WF, Talts KH, Sharrock NE. One hundred percent incidence of hemidiaphragmatic paresis associated with interescalene brachial plexus anesthesia as diagnosed by ultrasonography. Anesth Analg 1991;72:498-503.

25.- Urmey WF, McDonald M. Hemidiaphragmatic paresis durin interscalene brachial plexus block: effects on pulmonary function and chest wall mechanics. Anesth Analg 1992; 74:352-7.

26.- Riazi S, Carmichael N, Awad I, et al. Effect of local anaesthetic volume ( $20 \mathrm{vs} 5 \mathrm{ml}$ ) on the efficacy and respiratory consequences of ultrasound-guided interescalene brachial plexus block. Br J Anaesth 2008;101:549-56.

27.- Lee J-H, Cho S-H, Kim S-H, et al. Ropivacaine for ultrasound-guided interscalene block: $5 \mathrm{ml}$ provides similar analgesia but less phrenic nerve paralysis than 10ml. Can J Anaesth 2011; 58:1001-6.

28.- Stunder O, Meissnitzer M, Brummett CM, et al. Comparison of tissue distribution, phrenic nerve involvement, and epidural spread in standard-vs low-volume ultrasound-guided interscalene plexus block using contrast magnetic resonance imaging: a randomized, controlled trail. Br J Anaesth 2016;116:405-12.

29. Thackeray EM, Swenson JD, Gertsch MC, et al. Diaphragm function after interscalene brachial plexus block: a double-blind, randomized comparison of 0,25\% and 0,125\% bupivacaine. J Shoulder Elbow Surg 2013; 22:381-6.

30.- Wong AK, Keeney LG, Chen L, et al. Effect of local anesthetic concentration ( $0,2 \%$ vs $0,1 \%$ ropivacaine) on pulmonary function, and analgesia after ultrasound-guided interscalene brachial plexus block: a randomized controlled study. Pain Med 2016;17:2397-403.

31.- Palhais N, Brull R, Kern C, et al. Extrafascial inyection for interscalene brachial plexus block reduces respiratory complications compared with a conventional intrafascial inyection: a randomized, controlled, double-blind trial. $\mathrm{Br} \mathrm{J}$ Anaesth 2016;116:531-7.

32.- Tran DQ, Elgueta MF, Aliste J, et al. Diaphragm-sparing nerve blocks for shoulder surgery. Reg Anesth Pain Med 2017;42:32-8.

33.- Tran DQ, Layera S, Bravo D, Cristi-Sanchéz I, Bermudéz L, Aliste J. Diaphragm.sparing nerve blocks for shoulder surgery, revisited. Reg Anesth Pain Med 2020;45:73-78 
34.- Rawal N. American Society of Regional Anesthesia and pain Medicine 2010 Gaston Labat Lecture: perineural catheter analgesia as a routine method ambulatory surgery-effective but unrealistic. Regional Anesthesia and Pain Medicine 2012;37:72-8.

35.- Fredrickson MJ, Leightley P, Wong A. An analysis of 1505 consecutive patients receiving continuous interscalene analgesia at home: a multicentre prospective safety study. Anaesthesia 2016;71:373-379.

36.- Marhofer P, Anderl W, Heuberer P. A retrospective analysis of 509 consecutive interscalene catheter insertions for ambulatory surgery. Anaesthesia 2015;70:41-46.

37.- Exparel Parsipanny FDA approval data:. https:/www.accessdata.fda.gov/drugsatfda_docs/ label/2018/022496s9lbl.pdf.

38.- Muñoz-Leyva F, Cubillos J, Chin KJ. Managing rebound pain after regional anesthesia. Korean J Anesthesiol 2020:Aug 10. doi: 10.4097/kja.20436

39.- Lavand'homme P. Rebound pain after regional anesthesia in the ambulatory patient. Curr Opin Anaesthesiol 2018;31:6.

40.- Behr A, Freo U, Ori C, Westermann B, Alemanno F. Buprenorphine added to levobupivacaine enhances postoperative analgesia of middle interscalene brachial plexus block. Journal of Anesthesia 2012;26:746-51.

41.- Alemanno F, Ghisi D, Fanelli A, et al. Tramadol and 0,5\% levobupivacaine for single-shot interscalene block: effects on postoperative analgesia in patients undergoing shoulder arthroplasty. Minerva Anestesiologica 2012;78:291-6.

42.- Lee Ar, Yi H-W, Chung IS, et al. Magnesium added to bupivacaine prolongs the duration of analgesia after interscalene nerve block. Canadian Journal of Anesthesia 2012;59:21-7.

43.- Faria-Silva R, de Rezende DC, Ribeiro JM, et al. Association of clonidine and ropivacaine in brachial plexus block for shoulder arthroscopy. Brazilian Journal of Anesthesiology 2016;66:335-40.

44.- Watanabe K, Tokumine J, Yorozu T. Particulate-steroid betamethasone added to ropivacaine in interscalene brachial plexus block for arthroscopic rotator cuff repair improves postoperative analgesia. BMC Anesthesiology 2016;16:84.

45.- Desmet M, Braems H, Reynvoet M, et al. I.V. and perineural dexamethasone are equivalent in increasing the analgesic duration of a single-shot interscalene block with ropivacaine for shoulder surgery: a prospective, randomized, placebo-controlled study. British Journal of Anaesthesia 2013;111:445-52.

46.- Pehora C, Pearson AM, Kaushal A. Dexamethasone as an adjuvant to peripheral nerve block. Cochrane Database Syst Rev. 2017;9:11(11):CD0011770.

47.- Williams BA, Hough KA, Tsui BY. Neurotoxicity of adjuvants used in perineural anesthesia and analgesia in comparison with ropivacaine. Reg Anesth Pain Med 2011;36:225-30.

48.- Rosenfeld DM, Ivancic MG, Hattrup SJ. Perineural versus intravenous dexamethasone as adjuncts to local anaesthetic brachial plexus block for shoulder surgery. Anaesthesia 2016;71:380-388.

49.- Auyong DB, Yuan SC, Choi DS, et al. A double-blind randomized comparison of continuous interscalene, supraclavicular and suprascapular blocks for total shoulder arthroplasty. Reg Anesth pain Med 2017; 42:302-9.

50.- Kim BG, Han JU, song JH, et al. A comparison of ultrasound guides interscalene and supraclavicular blocks for post-operative analgesia after shoulder surgery. Acta Anaesthesiol Scand 2017; 61:427-35.

51.- Aliste J, Bravo D, Fernández D, et al. A randomized comparison between interscalene and small-volume supraclavicular blocks for arthroscopic shoulder surgery. Reg Anesth Pain Med 2018; 43: 590-5

52.- El-Boghdadly K, Chin KJ, Chan VWS. Phrenic Nerve Palsy and Regional Anesthesia for Shoulder Surgery: Anatomical, Physiologic, and Clinical Considerations. Anesthesiology 2017;127:173-91.

53.- Kim DH, Lin Y, Beathe JC, et al. Superior trunk block: a phrenic-sparing alternative to the interscalene block: a randomized controlled trial. Anesthesiology 2019;131:521-33.

54.- Abdallah FJ, Wijeysundera DN, Laupacis A. Subomohyoid anterior Suprascapular block versus Interscalene Block for Arthroscopic Shoulder Surgery: A multicenter Randomized Trial. Anesthesiology 2020;132:839-53. 
55.- Lee SM, Park SE; Nam YS. Analgesic effectiveness of nerve block in shoulder arthroscopy: Comparison between interscalene, suprascapular and axillary nerve blocks. Knee Surg Sports Traumatol Arthrosc 2012;20:2573-8.

56.- Dhir S, Sondekoppan RV, Sharma R. A comparison of combined suprascapular and axillary nerve blocks to interscalene nerve block for analgesia in arthroscopic shoulder surgery: An equivalence study. Reg Anesth Pain Med 2016;41:564-71.

57.- Neuts A, Stessel B, Wouters PF. Selective suprascapular and axillary nerve block versus interscalene plexus block for pain control after arthroscopic shoulder surgery: A noninferiority randomized parallel-controlled clinical trial. Reg Anesth Pain Med 2018;43:738-44.

58.- Aliste J, Bravo D, Layera S, et al. Randomized comparison between interscalene and costoclavicular blocks for arthroscopic shoulder surgery. Reg Anesth Pain Med 2019;44:472-7.

59.- Brown DL, Cahill DR, Bridenbaugh LD. Supraclavicular nerve block: anatomic analysis of a method to prevent pneumothorax. Anesth Analg 1993;76:530-4.

60.- Perlas A, Lobo G, Lo N, Brull R. Ultrasound-guided supraclavicular block: outcome of 510 consecutive cases. Reg Anesth Pain Med 2009;34:171-6.

61.- Petrar SD, Seltenrich ME, Head SJ. Hemidiafragmatic paralysis following ultrasound-guided supraclavicular versus infraclavicular brachial plexus blockade: a randomized clinical trial. Reg Anesth Pain Med 2015;40:133-138.

62.- Park SK, Lee SY, Kim WH. Comparison of Supraclavicular and Infraclavicular Brachial Plexus Block: A Systemic review of randomized controlled Trials. Anesth Analg 2017;124:636-44.

63.- Dhir S, Brown B, Mack P. Infraclavicular and supraclavicular approaches to brachial plexus for ambulatory elbow surgery: A randomized controlled observer-blinded trial. Journal of Clinical Anesthesia 2018;48:67-72.

64.- Chin KJ, Alakkad H, Adnikary SD, et al. Infraclavicular brachial plexus block for regional anaesthesia of the lower arm. Cochrane Database Syst Rev. 2013;8:CD005487.

65.- Araneda A, Miranda P, Corvetto M, et al. Retrospective analysis of adverse events associated with continuous peripheral nerve blocks at home during 2013-2014. Reg Anesth Pain Med 2015;40:e103-e104

66.- Leurcharusmee P, Elgueta MF, Tiyaprasertkul W. A randomized comparison between costoclavicular and paracoracoid ultrasound-guided infraclavicular block for upper limb surgery. Can J Anaesth 2017;64:617-625.

67.- Songthamwat B, Karmakar MK, Li JW. Ultrasound-Guided Infraclavicular Brachial Plexus Block: Prospective Randomized Comparison of the lateral Sagittal and Costoclavicular Approach. Reg Anesth Pain Med 2018;43:825-831

68.- Christophe JL, Berthier F, Boillot A, et al. Assessment of topographic brachial plexus nerves variations at the axilla using ultrasonography. Br J Anaesth 2009;100:606-612

69.- Schroeder LE, Horlocker TT, Schroeder DR. The efficacy of axillary block for surgical procedures about the elbow. Anesth Analg 1996;83:747-51.

70.- Chin KJ, Alakkad H, Cubillos JE. Single, double or multiple-injection techniques for non-ultrasound guided axillary brachial plexus blocks in adults undergoing surgery of the lower arm. Cochrane Database Syst Rev. 2013;8:CD003842.

71.- Chan VW, Perlas A, McCartney CJ. Ultrasound guidance improves success rate of axillary brachial plexus block. Can J Anaesth 2007;54:176-82.

72.- Choi S, McCartney CJ. Evidence Base for the Use of Ultrasound for Upper Extremity Blocks: 2014 Update. Reg Anesth Pain Med 2016;41:242-50.

73.- Sunderland S, Yarnold CH, Head SJ. Regional versus General Anesthesia and the Incidence of Unplanned Health Care Resource Utilization for Postoperative Pain after Wrist Fracture Surgery. Reg Anesth Pain Med 2016;41:22-27. 
Proximal

Distal

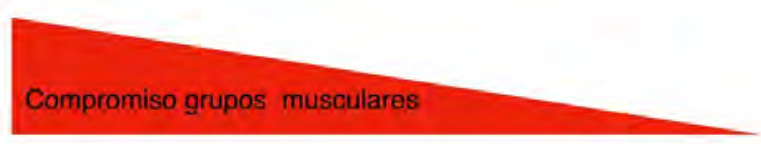

Selectividad, Seguridad 